\title{
Caracterización de la latencia invernal de cepas antiguas de vid (Vitis vinifera L.) País, Gros Colman y Torrontés Riojano, establecidas en el desierto de Atacama, Chile
}

\author{
Characterization of winter dormancy of ancient grapevine variety (Vitis vinifera $L$.) \\ País, Gros Colman y Torrontés Riojano, established Atacama desert, Chile
}

\author{
Ingrid Poblete $^{1 *}$, Patricio Hinrichsen ${ }^{2}$, Alejandro Riquelme ${ }^{3}$ y Manuel Pinto $^{2}$
}

\section{RESUMEN}

El desierto de Atacama presenta inviernos con altas temperaturas en el día, condición que limita la brotación y producción de especies frutales de hoja caduca; sin embargo, en esta zona se han encontrado plantas antiguas de vid, relictos de explotaciones vitivinícolas, las cuales han sobrevivido en este desierto absoluto, tolerando la alta fluctuación térmica invernal. Estas plantas constituyen un material genético adaptado a condiciones de desierto extremo, por lo cual es necesario caracterizar la latencia invernal de estas plantas, para su utilización productiva en zonas desérticas. Se analizaron seis accesiones: la cepa País de las localidades de Codpa, Suca, Pica, Cauquenes; la cepa Torrontés Riojano de Huaviña y Gros Colman de la Huayca (Canchones), quienes se establecieron en la Pampa del Tamarugal, Región de Tarapacá. Se llevaron a efecto muestreos entre abril y septiembre. Se evaluaron el número de días para alcanzar el $90 \%$ de brotación, tasa de brotación, brotación máxima, la concentración de $\mathrm{H}_{2} \mathrm{O}_{2}$ y la actividad de la CAT en las yemas. Los resultados indicaron que la accesión de Canchones requirió el menor número de días para alcanzar el $90 \%$ de brotación, presentando una respuesta similar en todo el período. Las accesiones de Codpa y Cauquenes tuvieron una significativa disminución en el número de días (90\% brotación) con una acumulación de 36,2 UF, mientras que en la de Suca se presentó con 74,6 UF. La accesión de Cauquenes y aquellas del norte se diferenciaron en la tasa de brotación. A nivel bioquímico, la accesión de Canchones presentó baja concentración de $\mathrm{H}_{2} \mathrm{O}_{2}$ con una acumulación de 36,2 UF, a diferencia de Codpa, Pica, Suca y Cauquenes, por otro lado, con 81,4 UF la accesión de Canchones tuvo la concentración más alta y Suca la menor. Solo hubo diferencias entre accesiones en la actividad de la CAT con 44,9 UF, en la que la accesión de Pica evidenció la actividad más alta. Se puede concluir que la accesión de Canchones no presenta una marcada endolatencia. Las accesiones de Codpa, Pica, Huaviña y Suca presentan una respuesta positiva y diferencial al frío nocturno invernal de la Pampa del Tamarugal, siendo Codpa la más temprana y Suca la más tardía. Existen diferencias entre accesiones a nivel bioquímico; sin embargo, no es posible establecer una relación entre la concentración de $\mathrm{H}_{2} \mathrm{O}_{2}$ y actividad de la CAT con la salida de la endolatencia en estas condiciones, siendo necesario nuevas investigaciones. Estas accesiones podrían ser una alternativa interesante para zonas cálidas.

Palabras clave: latencia, Vitis vinifera, desierto.

\section{ABSTRACT}

The Atacama desert presents winters with high temperatures during the day, condition that limits the sprouting and production of deciduous fruit trees. However, in this zone there have been found ancient grapevine, relict wine farms, which have survived in this absolute desert, whith tolerates hight thermal fluctuation. These plants are a genetic material adapted to extreme desert conditions, so it is necessary to characterize the winter dormancy of these plants for productive use in the desert areas. Six accessions accessions were analysed: The País wine variety from Codpa, Suca, Pica, Cauquenes; Torrontés Riojano wine variety from Huavina and Gros Colman from La Huayca (Canchones) locations, which are settled in the Pampa of Tamarugal, Tarapaca Region. The sampling was conducted between April and September. The number of days to reach $90 \%$ of sprouting, rate of sprouting, budding maximum, $\mathrm{H}_{2} \mathrm{O}_{2}$ concentration and the activity of $\mathrm{CAT}$ in the bud were evaluated. The results indicated that the accession of Canchones required the least number of days to reach $90 \%$ of budding, presenting a similar response throughout the period. Codpa and Cauquenes accessions had a significant decrease in the number of days to reach $90 \%$ sprouting with an accumulation of 36.2 UF, while in Suca arose to 74.6 CU. The accession of Cauquenes and those from the North differed regarding the rate of sprouting. At the Bioquemical level, accession of Canchones presented a low concentration of $\mathrm{H}_{2} \mathrm{O}_{2}$ with accumulation of $36.2 \mathrm{CU}$,

\footnotetext{
Universidad Arturo Prat, Facultad de Recursos Naturales Renovables, PO Box 121, Iquique, Chile.

2 Instituto de Investigaciones Agropecuarias, INIA Centro de Investigación La Platina, Santiago, Chile.

3 Centro de Estudios Avanzados en Fruticultura CEAF, Rengo, Chile.

* Autor para correspondencia: ingrid.poblete@unap.cl
}

Fecha de Recepción: 12 Abril, 2016.

Fecha de Aceptación: 2 Agosto, 2016. 
unlike Codpa, Pica, Suca and Cauquenes, on the other hand, with 81.4 CU, accession of Canchones had the highest concentration and Suca accession the lowest. There were only differences between accessions in activity of CAT with 44.9 CU in which Pica accession showed the highest activity. It can be concluded that accession of Canchones does not present a markerd endodormancy. Codpa, Pica, Suca and Huaviña accessions present a positive and differential response to chilling winter night of Pampa del Tamarugal, being Codpa the earliest and Suca the latest. There are differences at the biochemical level, however it is not possible to establish a relationship between the concentration of $\mathrm{H}_{2} \mathrm{O}_{2}$ and CAT activity in buds, with the ending of endodormancy under these conditions, further research are still needed in this regard. These accessions could be an interesting alternative for warn areas.

Key words: dormancy, Vitis vinifera, desert.

\section{Introducción}

La vid (Vitis vinifera L.) por ser originaria de zonas templadas, durante el período de latencia invernal debe acumular una determinada cantidad de horas frío (HF), para poder brotar y florecer uniformemente en la primavera siguiente. El estado de latencia, dependiendo de la causa de inhibición, se ha dividido en tres etapas: paralatencia en la que las yemas no brotan por la inhibición de otro órgano, endolatencia limitada por condiciones internas de la yema, es la etapa que requiere de la acumulación de las HF y ecolatencia en la que las yemas no brotan por condiciones ambientales restrictivas (Lang, 1987). La cantidad de HF depende en gran medida de la variedad, las que son numerosas en esta especie y cuyos requerimientos fluctúan entre 150 y 1.500 horas (Pinto et al., 2008). Cuando estos requerimientos no se cumplen, se manifiestan desórdenes fisiológicos como brotación tardía y desuniforme (Vergara y Pérez, 2010), floración irregular y extendida, escaso follaje y disminución de rendimiento (Campoy et al., 2010; Elloumi et al., 2013; Ghrab et al., 2014). Para el cálculo de horas frío tradicionalmente se ha usado el método directo de conteo de horas entre $0^{\circ} \mathrm{C}$ y $7^{\circ} \mathrm{C}$, el cual otorga la misma contribución de frío a todas las temperaturas comprendidas en este intervalo. En condiciones subtropicales, las temperaturas máximas sobrepasan, durante el día, considerablemente el umbral de $7{ }^{\circ} \mathrm{C}$, existiendo una gran amplitud térmica diaria invernal, presentándose temperaturas máximas diarias por sobre los $25^{\circ} \mathrm{C}$ (Erez et al., 1993; Lanino, 2004), situación que afecta la salida de la endolatencia (Campoy et al., 2011). Bajo estas condiciones sería más adecuado usar el concepto de unidad frío (UF) (Pérez et al., 2008a), que considera la eficiencia de la temperatura en el proceso de acumulación de frío (Richardson et al., 1974).

En las condiciones de desierto, como es el caso del extremo norte de Chile, específicamente en localidades de las regiones de Tarapacá y de Arica y
Parinacota, existen plantas antiguas de vid, que según los lugareños poseen más de 100 años de edad. Estas plantas, debiso al tiempo que llevan en estas regiones, es probable que se hayan aclimatado a los inviernos caracterizados por altas temperaturas diurnas y bajas temperaturas nocturnas (Lanino, 2004). De acuerdo con lo anterior, se podría postular que estas plantas poseen un bajo requerimiento de frío invernal, lo que correspondería a un rasgo adaptativo (Londo y Johnson, 2014) y, por tanto, es posible que en estas condiciones de aridez, exista un efecto positivo de las bajas temperaturas nocturnas para inducir la salida de la endolatencia, similar a lo que se ha observado en condiciones subtropicales, en donde la predominancia de temperaturas moderadas diurnas y bajas temperaturas nocturnas diarias, favorecen el proceso de acumulación de frío invernal. Así, en Prunus persica y Actinidia deliciosa la incidencia de temperaturas diurnas, entre $13{ }^{\circ} \mathrm{C}$ y $15^{\circ} \mathrm{C}$, ejercen un fuerte sinergismo con temperaturas nocturnas inferiores a $12{ }^{\circ} \mathrm{C}$, en el proceso de la salida de la endolatencia (Warrington y Stanley, 1986; Erez, 1995); es decir, permitirían fijar el efecto del frío en la yema, según lo propuesto en el modelo dinámico (Pérez et al., 2008a).

Por otro lado, si bien se conocen los efectos externos que producen las temperaturas invernales sobre la brotación de las plantas, es muy poco lo que se sabe sobre los cambios internos que la inducen, entre estos los cambios bioquímicos. $\mathrm{Al}$ respecto, se ha postulado que las especies oxígeno reactivas, juegan un rol fundamental en la salida de la endolatencia (Meitha et al., 2015), en especial el peróxido de hidrógeno $\left(\mathrm{H}_{2} \mathrm{O}_{2}\right)$ (Pérez y Lira, 2005; Pérez et al., 2007; Pinto et al., 2008). Este metabolito, cuya concentración tiende a aumentar a medida que se incrementa la acumulación de frío (Zhi-You et al., 2003), se indica que puede actuar como molécula señal en la salida de la endolatencia, (Pérez y Lira, 2005; Pérez et al., 2007). A la vez, como el $\mathrm{H}_{2} \mathrm{O}_{2}$ es una molécula que causa daño celular, existe un sistema de protección dado por 
un grupo de enzimas antioxidantes como la catalasa (CAT) (Pérez y Lira, 2005; Vergara et al., 2012), la ascorbato peroxidasa y la glutation reductasa (Noctor y Foyer, 1998; Halaly et al., 2008; Vergara et al., 2012). Estas enzimas degradan el $\mathrm{H}_{2} \mathrm{O}_{2}$ a oxígeno y otros productos secundarios para evitar el daño y finalmente la muerte celular (Asada, 1992). De este grupo de enzimas se ha demostrado que la CAT parece jugar un rol principal en vid, se ha observado que la actividad de esta enzima aumentó durante el período de endolatencia alcanzando un máximo y posteriormente disminuyó a menos de un tercio de la máxima actividad (Pérez y Lira, 2005), similar a lo observado previamente por Nir et al. (1986).

Así también, la aplicación de cianamida hidrogenada el principal producto que se utiliza para estimular la brotación en zonas con insuficiente frío invernal (Vergara y Pérez, 2010), ejerce su acción por medio de la inhibición de la catalasa, lo que ocasiona el aumento en la concentración de $\mathrm{H}_{2} \mathrm{O}_{2}$ (Pérez y Lira, 2005; Halaly et al., 2008; Pérez et al., 2008b). De este modo, si existen variaciones en los requerimientos de frío entre diferentes accesiones de vid, es posible que existan diferencias en las concentraciones de $\mathrm{H}_{2} \mathrm{O}_{2}$ y en la actividad de la catalasa, entre accesiones con diferentes requerimientos de frío.

Basado en lo anteriormente expuesto, el objetivo de este estudio fue caracterizar la latencia invernal de accesiones antiguas de vid, en las condiciones climáticas del norte del desierto de Atacama, el que posee altas temperaturas diurnas y bajas temperaturas nocturnas.

\section{Materiales y Métodos}

\section{Material vegetal}

Se emplearon cinco accesiones procedentes de diferentes localidades de la Región de Tarapacá y de Arica y Parinacota: La Huayca, Canchones $\left(20^{\circ}\right.$

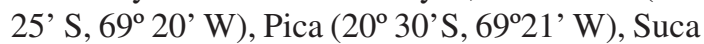

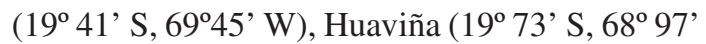
W) y Codpa $\left(18^{\circ} 50^{\prime} \mathrm{S}, 69^{\circ} 45^{\prime} \mathrm{W}\right)$, como también la variedad País procedente de Cauquenes en el sur de Chile ( $35^{\circ} 58^{\prime} \mathrm{S} 72^{\circ} 21^{\prime} \mathrm{W}$ ). Las accesiones de Pica, Suca y Codpa fueron identificadas mediante análisis genético, como la variedad País y de Canchones como la variedad Gros Colman (Poblete et al. 2011), En el año 2012 la accesión de Huaviña fue identificada por el INRA de Montpellier como Torrontés Riojano. Estas plantas fueron propagadas por estacas y plantadas en septiembre del 2004 en la Pampa del Tamarugal, en la Estación Experimental Canchones de la Universidad Arturo Prat $\left(20^{\circ}\right.$ $\left.25^{\prime} \mathrm{S}, 69^{\circ} 20^{\prime} \mathrm{W}\right)$. Se establecieron en sistema de espaldera simple a una distancia de plantación de $2 \mathrm{~m}$ entre hileras y $1,5 \mathrm{~m}$ sobre la hilera, con riego por goteo. La investigación se llevó a efecto en el año 2007 en las condiciones climáticas de la Pampa del Tamarugal (Tabla 1).

Para caracterizar la latencia invernal de cada accesión se colectaron estacas que comprendían entre el $3^{r}$ y 12 nudos cada 14 días, a partir del 13 de abril y hasta el 14 de septiembre. De las estacas colectadas en cada muestreo se extrajeron las yemas del nudo 10 al 12, para los análisis bioquímicos, las que fueron envueltas en papel de aluminio,

Tabla 1. Características climáticas de la Pampa del Tamarugal, Región de Tarapacá. 2007

\begin{tabular}{|c|c|c|c|c|c|}
\hline Mes & $\mathrm{T}^{\mathrm{o}}$ Máxima $\left({ }^{\circ} \mathrm{C}\right)$ & $\mathrm{T}^{\mathrm{o}}$ Mínima $\left({ }^{\circ} \mathrm{C}\right)$ & $\mathrm{HF}\left(0-7^{\circ} \mathrm{C}\right)$ & UF (*) & DG (base $10^{\circ} \mathrm{C}$ ) \\
\hline Enero & 32,5 & 13,1 & & & 351,0 \\
\hline Febrero & 31,3 & 13,5 & & & 326,0 \\
\hline Marzo & 29,9 & 8,5 & & & 342,0 \\
\hline Abril & 31,1 & 6,5 & & & 262,0 \\
\hline Mayo & 29,9 & 2,6 & 209,0 & 32,0 & 223,0 \\
\hline Junio & 29,2 & 2,0 & 233,5 & 34,3 & 184,0 \\
\hline Julio & 29,6 & 0,2 & 225,0 & 14,0 & 197,0 \\
\hline Agosto & 28,8 & 1,1 & 258,0 & 77,5 & 225,0 \\
\hline Septiembre & 31,5 & 2,4 & 196,5 & 16,5 & 240,0 \\
\hline Octubre & 31,9 & 3,4 & & & 268,0 \\
\hline Noviembre & 32,4 & 5,0 & & & 333,0 \\
\hline Diciembre & 31,8 & 6,4 & & & 387,0 \\
\hline
\end{tabular}

(*): Según Richardson et al. (1974). HF y UF solo se indican para el período de acumulación de frío. 
sumergidas en nitrógeno líquido y almacenadas a $-80^{\circ} \mathrm{C}$ hasta su análisis.

\section{Inducción forzada de la brotación y caracterización de esta}

Para inducir la brotación se emplearon diez estacas de un nudo por repetición, las que el mismo día del muestreo, se insertaron en una lámina de poliestireno y se mantuvieron flotando en agua destilada en una bandeja de pvc $(55 \times 15 \times 10 \mathrm{~cm})$ hasta su brotación. Durante la inducción de la brotación, la temperatura en la cámara fue mantenida a $25^{\circ} \mathrm{C} \pm 1,5^{\circ} \mathrm{C}$, y con 12 horas de fotoperíodo, dado por lámparas que proveyeron una intensidad de radiación PAR de $100 \mu \mathrm{mol} \mathrm{m}{ }^{-2} \mathrm{~s}^{-1}$ a nivel de las estacas.

La caracterización de la brotación se efectuó mediante determinación visual tres veces por semana, considerando el momento de inicio de brotación aquel en que las yemas presentaron el estado de punta verde y el de término cuando la brotación se estabilizó. De estas observaciones se obtuvieron las siguientes variables: número de días para alcanzar el $90 \%$ de brotación; tasa de brotación la que se calculó en base al número total de yemas brotadas, dividido por el número de días requeridos para alcanzar esta brotación y brotación máxima, determinada en relación al total de yemas brotadas por accesión.

\section{Determinación de las necesidades de frío y análisis bioquímicos en las yemas}

Las necesidades de frío por accesión se determinaron en base a las HF y las UF registradas en la Estación Experimental Canchones. Las HF fueron establecidas mediante la suma de las temperaturas comprendidas entre $0^{\circ} \mathrm{C}$ y $7^{\circ} \mathrm{C}$ y las UF obtenidas de acuerdo con el modelo de Richardson et al. (1974). Las variables bioquímicas se analizaron en seis fechas de muestreo: 26 de mayo, 8 de junio, 22 de junio, 19 de julio, 3 de agosto y 17 de agosto, correspondiente a $0 ; 36,2 ; 44 ; 74,6 ; 81,4 ; 119,3 \mathrm{UF}$, respectivamente. En cada muestreo se colectaron 18 yemas, se empleó una yema para el análisis de $\mathrm{H}_{2} \mathrm{O}_{2}$ y dos yemas para la actividad de la CAT, con tres repeticiones bioquímicas para cada caso.

La concentración de $\mathrm{H}_{2} \mathrm{O}_{2}$ se determinó de acuerdo con Hung y Kao (2004). Para esto, se empleó la solución de extracción compuesta por ácido tricloroacético (TCA) al 0,1\%. Para la determinación de la concentración de $\mathrm{H}_{2} \mathrm{O}_{2}$ se midió la absorbancia del sobrenadante a $390 \mathrm{~nm}$ y este valor menos el blanco, el que consistió en una solución compuesta por TCA, buffer fosfato y KI, se empleó en el cálculo de la concentración, utilizando una curva de calibración con diluciones de $\mathrm{H}_{2} \mathrm{O}_{2}$ estándar. El resultado se expresó en $\mu \mathrm{g} \mathrm{g}{ }^{-1}$ de PF.

La actividad de la CAT se evaluó en extractos de yemas. Para esto, dos yemas de aproximadamente $90 \mathrm{mg}$ cada una, se molieron en nitrógeno líquido, posteriormente se agregó $1 \mathrm{~mL}$ de solución tampón de fosfato potásico (TFP) a $90 \mathrm{mM}$ (pH 7,8), $1 \mathrm{mM}$ de EDTA, $8 \%$ de glicerol y polyvinylpoypyrrolidona (PVPP) (0,3 g por g de tejido). Esta determinación se efectuó de acuerdo con Beers y Sizer (1952) para lo cual la solución de reacción empleada fue: $100 \mathrm{mM}$ buffer fosfato (pH 7,0), 0,1 $\mu$ M EDTA, $20 \mathrm{mM} \mathrm{H}_{2} \mathrm{O}_{2}$. Se monitoreó el descenso de $\mathrm{H}_{2} \mathrm{O}_{2}$ por la medición de la absorbancia a $240 \mathrm{~nm}$ desde el momento en que se adicionó el $\mathrm{H}_{2} \mathrm{O}_{2}$ hasta 1 min más tarde. La diferencia en absorbancia $(\triangle A 240)$ fue dividida por el coeficiente de extinción molar del $\mathrm{H}_{2} \mathrm{O}_{2}\left(36 \mathrm{M}^{-1} \mathrm{~cm}^{-1}\right)$ y la actividad de la enzima fue expresada como $\mu \mathrm{mol}$ de $\mathrm{H}_{2} \mathrm{O}_{2}$ $\mathrm{min}^{-1} \mathrm{mg}^{-1}$ proteína, para lo que se determinó la concentración de proteína total en la fracción soluble del extracto de yema mediante el método de Bradford (1976), y se utilizó como proteína patrón, suero de albúmina de bovino. Se consideró $50 \mu \mathrm{L}$ del extracto de yema, $1 \mathrm{~mL}$ de reactivo de Bradford, se incubó por 10 min en oscuridad y se midió la absorbancia a $595 \mathrm{~nm}$.

\section{Diseño experimental y análisis estadístico}

Se utilizó un diseño completamente al azar, empleando tres repeticiones por tratamiento y la unidad experimental correspondió a diez estacas uninodales para brotación forzada y una y dos yemas para el análisis de $\mathrm{H}_{2} \mathrm{O}_{2}$ y actividad de la CAT, respectivamente. Los resultados se sometieron a un análisis de varianza (ANDEVA) y las diferencias se separaron mediante la prueba de comparación medias de Tukey $(\mathrm{p} \leq 0,05)$. Cuando correspondió se realizó análisis de regresión. El programa computacional utilizado fue el INFOSTAT® versión 2007 p. El análisis de las pendientes de las curvas de regresión se realizó mediante el programa SAS "Multiple Comparisons of Slopes" con el procedimiento GLM SUMMARY. 


\section{Resultados y Discusión}

\section{Efecto de la acumulación de frío sobre la precocidad de brotación}

La accesión de Canchones (barra negra, Figura 1) en general no cambia su comportamiento de brotación a lo largo de la temporada invernal. Esta accesión es la que menos responde al frío y bajo las condiciones de la presente investigación, prácticamente no presentó endolatencia. Debido a lo anterior, en este caso en terreno, las yemas se encontrarían en ecolatencia inhibidas de brotar no por factores endógenos sino por condiciones ambientales desfavorables como temperaturas inferiores a $0^{\circ} \mathrm{C}$ y/o restricción hídrica. Sin acumulación de frío (0 UF), el número de días requeridos para un $90 \%$ de brotación fluctuó entre 28 y 42 días, no existiendo diferencias entre las accesiones. Este número de días, a excepción de la accesión de Canchones, fue inferior a aquel encontrado para todas las accesiones cuando la acumulación de frío fue de 6,2 UF (11 de mayo), momento en el que la accesión se diferencia de Suca, la cual requiere 55 días para alcanzar este nivel de brotación. Esto indica que en todas las accesiones, las yemas muestreadas antes del 15 de abril, se encontraban en paralatencia; es decir, inhibidas por acción de la dominancia apical y presencia de hojas (Cook et al., 2005). Con una acumulación de 29 UF (25 de mayo) el número de días para alcanzar el $90 \%$ de brotación varió entre 32 días en la accesión de Canchones, que como ya se ha indicado presenta baja variación en su brotación y un máximo de 61 días en la accesión de Suca. Esto demuestra que a esta fecha en casi todas las accesiones las yemas estaban en endolatencia. Lo anterior, a pesar que las plantas de todas las accesiones, se encontraban con la mayoría de sus hojas verdes. Lo expuesto, tiene relación con lo informado por Cook et al. (2005) quienes manifiestan que las yemas entran en endolatencia independientemente de la presencia de hojas y, por otro lado, Taylor et al. (1984) señalan que la presencia de hojas contribuye a aumentar el ácido abscísico (ABA) en las yemas, el cual cumpliría una función clave en la entrada en endolatencia (Parada et al., 2016).

La significativa disminución del número de días para alcanzar el $90 \%$ de brotación que se observa después del 25 de mayo en las accesiones de Cauquenes y Codpa, se debería al término de la endolatencia en estas accesiones (Figura 1) y a la entrada en la ecolatencia. Así, por ejemplo, en la accesión de Cauquenes que hasta el 25 de mayo se demoró 44 días para romper la endolatencia, el 8 de junio fue de solo 24 días; es decir, a esta fecha con una acumulación de frío de 36,2 UF, en esta accesión el período para romper la endolatencia se reduce en 20 días. Una disminución similar se observó en la accesión de Codpa siendo esta de 25 días. En el caso de la accesión de Canchones, que como ya se dijo responde poco al frío, este decremento fue menor

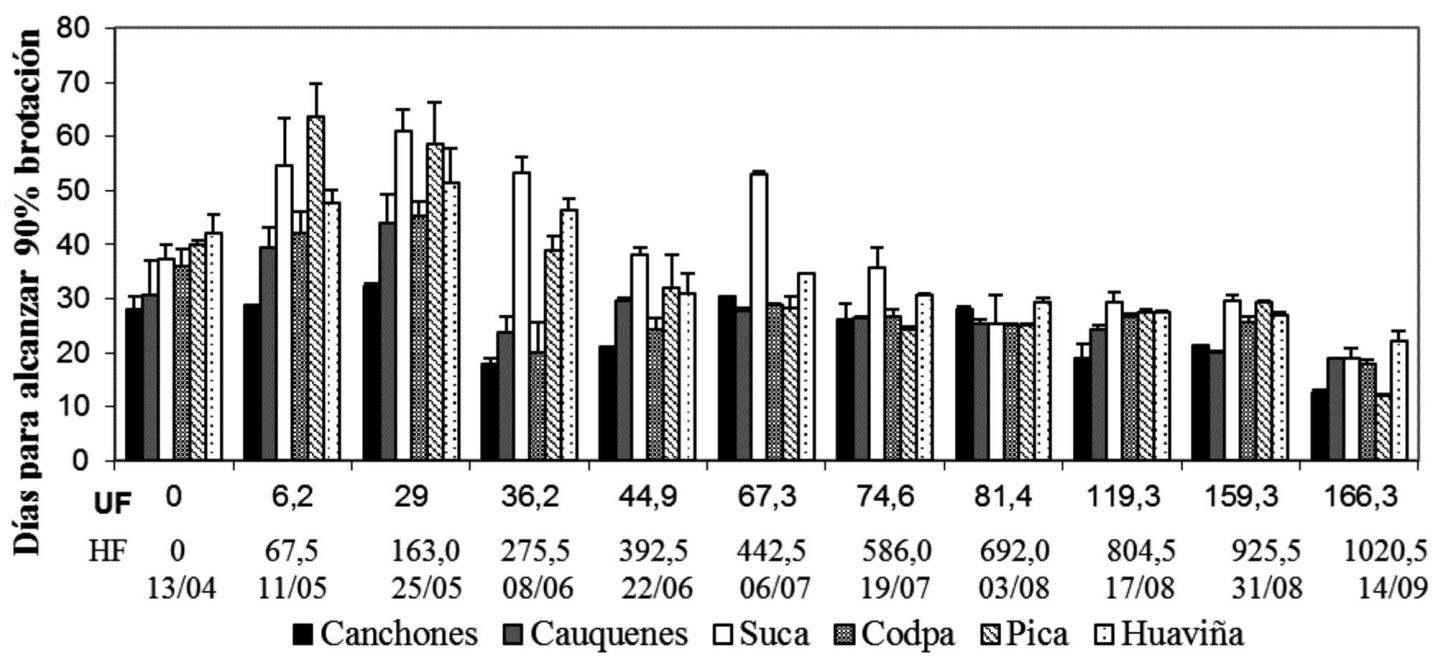

Figura 1. Número de días requeridos por cada accesión de vid, para alcanzar el $90 \%$ de brotación de sus yemas en cada fecha de muestreo. Líneas verticales sobre barras indican error estándar ( $\mathrm{p} \leq 0,05)$. 
de 14 días solamente. La reducción en el número de días para brotar antes señalada, indica que estas accesiones poseen un requerimiento de frío para salir de su endolatencia cercano a las 36 UF. En cambio en la accesión de Suca este requerimiento fue mucho mayor cercano a las 75 UF. En efecto, en esta accesión el término de la endolatencia se produjo el 19 de julio, un mes y medio más tarde que en las accesiones antes mencionadas. En el caso de la accesión de Pica y Huaviña, presentan un requerimiento de frío invernal intermedio de aproximadamente 45 UF.

Esta disminución en el número de días se debería al efecto del frío nocturno en las yemas, cuyas temperaturas mínimas en la zona fluctuaron entre 0,2 y $2,4^{\circ} \mathrm{C}$ en el período comprendido entre el 26 al 31 de mayo y entre 0,9 y $2,5^{\circ} \mathrm{C}$, en el período entre el 1 al 8 de junio (Datos Estación Meteorológica de Estación Experimental Canchones), lo que puede corresponder a un rasgo adaptativo de estas plantas (Londo y Johnson, 2014).

El aumento de la brotación se debería al efecto del frío sobre procesos internos en las yemas como, por ejemplo, el flujo de carbohidratos solubles desde el tallo hacia el primordio (Marquat et al., 1999; Bonhomme et al., 2005). Durante la endolatencia se ha propuesto que se produce la hidrólisis del almidón y la oxidación del sorbitol los cuales dan origen a glucosa y fructosa, respectivamente; esto ocasiona la acumulación de sacarosa (Marquat et al., 1999; Ben Mohamed et al., 2010; Rubio et al., 2014; Rubio et al., 2016), como fuente de carbono, debido a la baja absorción en ese momento, lo que permite disponer de carbohidratos solubles para la brotación (Ben Mohamed et al., 2012).

\section{Efecto de la acumulación de las unidades frío sobre la tasa de brotación}

Existe una relación positiva entre la acumulación de las UF y la tasa de brotación en las diferentes accesiones (Figura 2). El mayor ajuste a la recta lo presentaron las accesiones de Cauquenes y Huaviña $\left(\mathrm{R}^{2}=0,84\right.$ y 0,82 , respectivamente) y el menor, las accesiones de Pica y Canchones $\left(\mathrm{R}^{2}=0,56\right.$ y 0,57, respectivamente). Estas últimas coinciden con las localidades que presentan las temperaturas más altas en el período invernal.
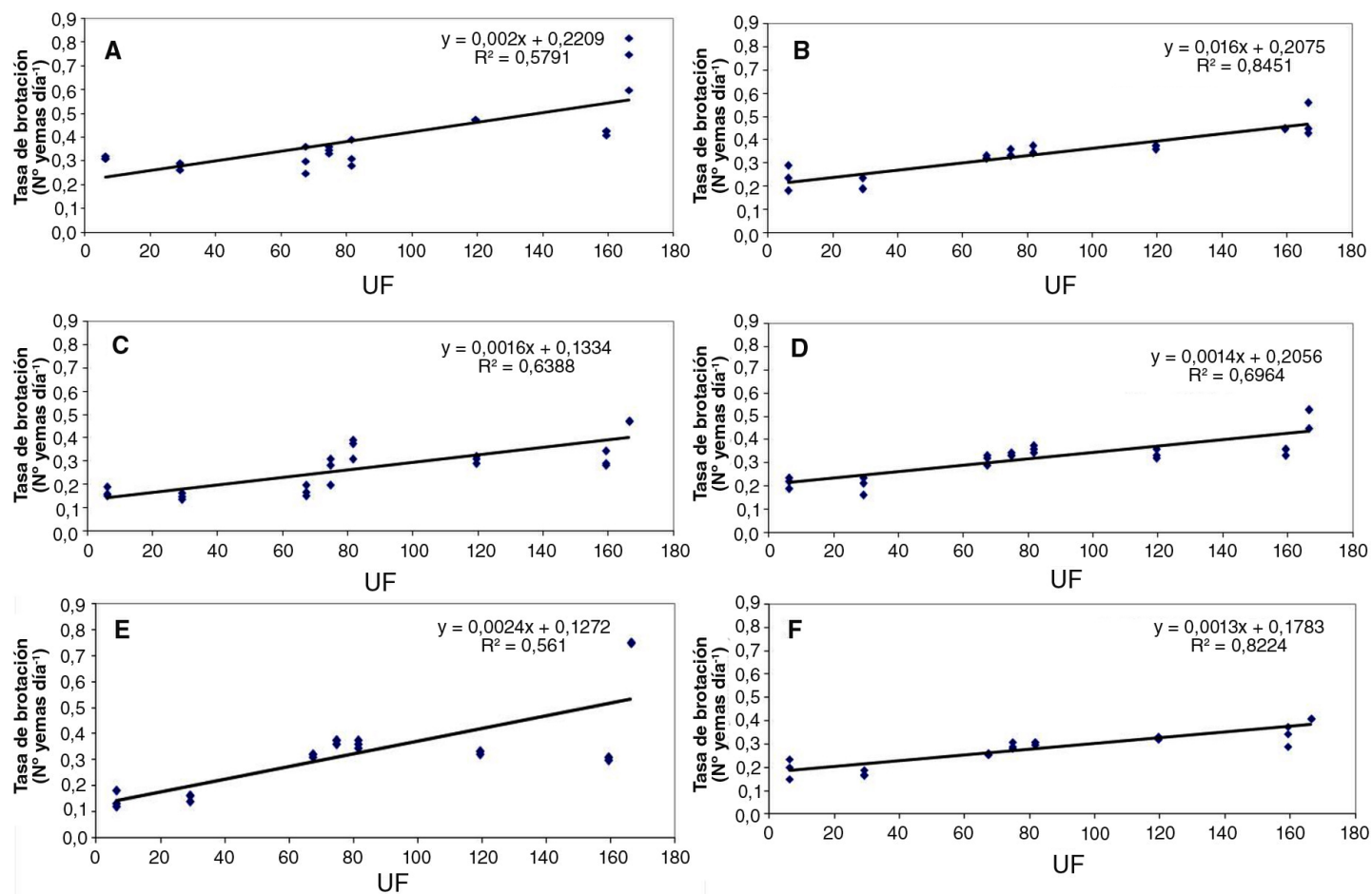

Figura 2. Relación entre la acumulación de UF y la tasa de brotación para las accesiones de: Canchones (A), Cauquenes (B), Suca (C), Codpa (D), Pica (E) y Huaviña (F), en condiciones de la Pampa del Tamarugal. 
Las tasas de brotación de la accesión de Cauquenes fue diferente $(\mathrm{p} \leq 0,05)$ a la tasa de brotación de todas las accesiones locales, lo anterior demuestra que esta accesión, introducida de la zona centro sur, presenta un comportamiento de brotación distinto con respecto a las accesiones locales, como respuesta a las condiciones invernales cálidas, denotando la adaptación de las accesiones del norte (Londo y Johnson, 2014).

Destaca la alta tasa de brotación de la accesión de Pica siendo casi el doble que la de la accesión de Codpa (Figura 2). Esto indica una gran variación en este carácter y, por lo tanto, una diferencia notable en la sensibilidad al frío nocturno entre accesiones genéticamente iguales, ya que ambas corresponden a la variedad País (Poblete et al., 2011).

\section{Brotación máxima alcanzada en yemas vid, considerando distintas fechas de acumulación de unidades frío}

Para todas las fechas de muestreo se encontró un porcentaje de brotación igual o superior al $80 \%$, la excepción la constituyó la accesión de Pica para la tercera fecha (11/05/07), en el que la brotación se estabilizó en un $60 \%$ (Figura 3). Los requerimientos de frío se consideran satisfechos cuando el $50 \%$ de las yemas es capaz de brotar (Hauagge y Cummins, 1991), y observando que para todas las fechas de muestreo siempre se superó este porcentaje, se podría afirmar que las accesiones bajo estas condiciones climáticas cumplen sus requerimientos de frío, debido a que al colocarlas en condiciones favorables se estimuló un alto porcentaje de brotación. Sin embargo, es importante destacar que este valor por sí solo no es relevante, ya que es necesario tener en cuenta el tiempo requerido para lograr esta brotación máxima. Esto último, de acuerdo con el modelo de Richardson et al. (1974), está asociado
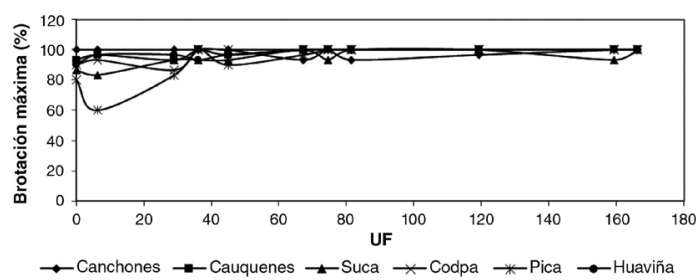

Figura 3. Efecto de la acumulación de UF sobre la brotación máxima en yemas de vid de diferentes accesiones, establecidas en la Pampa del Tamarugal. N.S. (Tukey, $\mathrm{p} \leq 0,05$ ). a la efectividad del frío recibido. Por lo tanto, la diferencia entre las accesiones radica en la tasa de brotación (Figura 2) y no en el porcentaje total de brotación, también observado previamente (Poblete et al. (2009).

\section{Relación entre la concentración de $\mathrm{H}_{2} \mathrm{O}_{2}$ y la brotación en vid}

El $\mathrm{H}_{2} \mathrm{O}_{2}$ en las yemas solo mostró diferencias $(\mathrm{p} \leq 0,05)$ en su concentración entre las accesiones, cuando se habían acumulado 36,2 UF, 44,9 UF y 81,4 UF (Figura 4).

Para el muestreo efectuado el 8 de junio $(36,2$ UF) la accesión de Canchones presentó la menor concentración de $\mathrm{H}_{2} \mathrm{O}_{2}$ respecto de las accesiones de Pica, Codpa, Cauquenes y Suca. Al respecto, es importante señalar que con esta acumulación de UF, las accesiones de Codpa y Cauquenes se encontraban en estado de salida de endolatencia. Por otro lado, en la accesión de Canchones que presenta baja variación en el tiempo (Figura 1), se produjo un aumento en la concentración de $\mathrm{H}_{2} \mathrm{O}_{2}$ a medida que se incrementaron las UF $(81,4)$ (Figura 4), concordante con lo expuesto por Kuroda et al. (2002), también observado en peral asiático (Kuroda et al., 2005) y duraznero (Zhi-You et al., 2003), condición que la diferenció de Suca, la que presentó la menor concentración. Sin embargo, en la accesión de Canchones a pesar del aumento de $\mathrm{H}_{2} \mathrm{O}_{2}$ con los días transcurridos, no tiene relación con la brotación, debido a que esta accesión no varió su comportamiento, al presentar un alto porcentaje de brotación en corto tiempo, durante todo el período de muestreo (abril-septiembre). Las diferencias encontradas entre la concentración de $\mathrm{H}_{2} \mathrm{O}_{2}$ para las distintas accesiones, no tuvo relación con la salida de

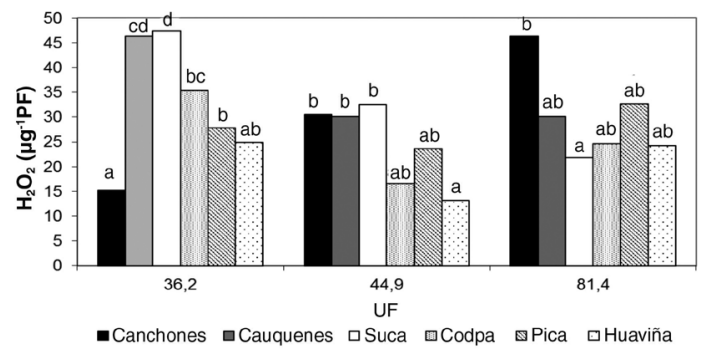

Figura 4. Efecto de las UF sobre la concentración de $\mathrm{H}_{2} \mathrm{O}_{2}$ en las yemas de diferentes accesiones de vid, cultivadas en condiciones de la Pampa del Tamarugal. Barras con diferentes letras para la misma acumulación de UF, indican diferencias significativas (Tukey, $\mathrm{p} \leq 0,05$ ). 
la endolatencia, difiriendo de lo informado por Pérez y Lira (2005) y Pérez et al. (2008b) para la variedad Thompson Seedless. Además, Kuroda et al. (2005) y Pérez et al. (2008b) encontraron que la aplicación exógena de esta molécula reprodujo parcialmente el efecto de inducción de la brotación, corroborando su efecto como molécula señal para la salida de la endolatencia. Por lo tanto, en este caso la respuesta observada se puede deber a un efecto transitorio del estrés oxidativo causado por el $\mathrm{H}_{2} \mathrm{O}_{2}$, como lo señalan Halaly et al. (2008), y que no fue posible, detectar; al efecto de las altas temperaturas sobre la concentración de $\mathrm{H}_{2} \mathrm{O}_{2}$, según lo indicado por ZhiYou et al. (2003); o bien una respuesta adaptativa diferente a las condiciones estresantes del extremo norte de Chile, lo que requiere mayor investigación.

\section{Efecto de la acumulación de unidades frío sobre la actividad de la CAT en yemas de vid}

En relación a la acción del frío sobre la actividad de la CAT en yemas de vid, solo se manifestaron diferencias entre las accesiones al momento en que las yemas habían acumulado 44,9 UF, es así como la actividad de la CAT en la accesión de Pica resultó ser más alta $\left(61,51 \mu \mathrm{mol} \mathrm{H}_{2} \mathrm{O}_{2}\right.$ min $^{-1}$ $\mathrm{mg}^{-1}$ proteína) y diferente de las otras accesiones. Por el contrario de lo observado, en la accesión de Canchones, aunque no manifestó diferencias con las otras accesiones, se presentó una menor actividad de la CAT $\left(3,52 \mu \mathrm{mol} \mathrm{H}_{2} \mathrm{O}_{2} \mathrm{~min}^{-1} \mathrm{mg}^{-1}\right.$ proteína) (Figura 5), concordando en este caso con una mayor concentración de $\mathrm{H}_{2} \mathrm{O}_{2}$ (CórdobaPedregosa et al., 2003; Zhi-You et al., 2003; Pérez et al., 2008b); no obstante, como se mencionó anteriormente la accesión de Canchones no presenta una clara endolatencia. La enzima CAT juega un rol fundamental en la salida de la endolatencia, la que disminuye su actividad a medida que avanza el período de enfriamiento (Nir et al., 1986; Pérez y Lira, 2005), acumulándose $\mathrm{H}_{2} \mathrm{O}_{2}$ (Zhi-You et al., 2003; Pérez et al., 2007), respuesta que también es provocada por muchos productos químicos que actúan sobre la brotación (Zhi-You et al., 2003; Pérez et al., 2008b). Se ha visto que no existe una clara relación entre la actividad de la CAT y la concentración de $\mathrm{H}_{2} \mathrm{O}_{2}$ (Pérez y Lira, 2005) lo cual, sugiere una actividad de otras enzimas que remueven el $\mathrm{H}_{2} \mathrm{O}_{2}$ como, por ejemplo, la participación de la ascorbato peroxidasa, glutation reductasa (Asada, 1992; Halaly et al., 2008) y que pueden ser importantes en la tolerancia al ataque oxidativo.

\section{Conclusiones}

En las condiciones del desierto de Atacama, la accesión de Canchones (Gros Colman) no presenta una marcada endolatencia, mostrando la capacidad de generar una alta brotación, en corto tiempo a lo largo del período invernal. Las accesiones de Codpa, Pica, Suca (País) y Huaviña (Torrontés Riojano) tienen una respuesta positiva y diferencial al frío nocturno invernal, siendo la accesión de Codpa la más temprana y Suca la más tardía. La accesión de Cauquenes (País) presenta una tasa de brotación distinta de las accesiones del norte. A nivel bioquímico, existen diferencias entre accesiones, sin embargo, no es posible establecer una relación entre la concentración de $\mathrm{H}_{2} \mathrm{O}_{2}$ y actividad de la CAT en las yemas con la salida de la endolatencia, pudiendo presentar estas accesiones; una respuesta diferente a aquellas de áreas templadas, considerando su adaptación a la fluctuación térmica invernal, siendo necesario nuevas investigaciones. Estas accesiones podrían ser una alternativa interesante para zonas cálidas.

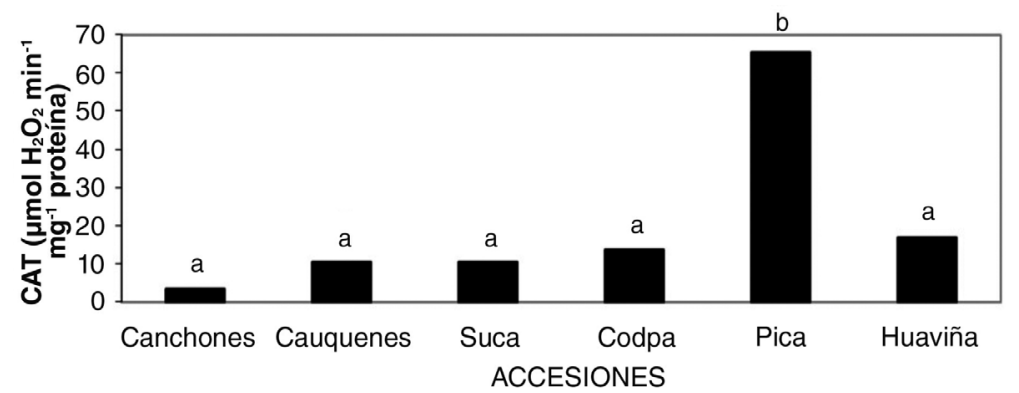

Figura 5. Actividad de la CAT en las yemas de diferentes accesiones de vid, cultivadas en condiciones de la Pampa del Tamarugal, con una acumulación de 44,9 UF. Barras con diferentes letras, indican diferencias significativas (Tukey, $\mathrm{p} \leq 0,05$ ). 


\section{Literatura Citada}

Asada, K.

1992. Ascorbate peroxidase-a-hydrogen peroxide-scavenging enzyme in plants. Physiol. Plant. 85: 235-241.

Beers, R.; Sizer. I.

1952. A spectrophotometric method for measuring the breakdown of hydrogen peroxide by catalase. J. Biol Chem. 195 (1): 133-40.

Bonhomme, M.; Rageau, R.; Lacointe, A.; Gendraud, M. 2005. Influences of cold deprivation during dormancy on carbohydrate contents of vegetative and floral primordia and nearby structures of peach buds (Prunus persica L. Batch). Scientia Horticulturae 105: 223-240.

Bradford, M.

1976. A rapid and sensitive method for the quantification of micrograms quantities of proteins utilizing the principle of protein-dye binding. Annual Biochem. 72: 248-254.

Campoy, J.; Ruiz, D.; Cook, N.; Allderman, L.; Egea, J.

2011. High temperatures and time to budbreak in low chill apricot "Palslegn" towards a better understanding of chill and heat requirements fulfillment. Scientia Horticulturae 129: 649-655.

Campoy, J.; Ruíz, D.; Egea, J.

2010. Effects of shading and thidiazuron + oil treatment on dormancy breaking, blooming and fruit set in apricot in a warm-winter climate. Scientia Horticulturae 125: 203-210.

Cook, N.; Bellen, A.; Cronjé, A.; De Wit, I.; Keulemans, W.; Van Den Putte, A.; Steyn, W.

2005. Freezing temperature treatment induces bud dormancy in "Granny Smith" apple shoots. Scientia Horticulturae 106: 170-176.

Córdoba-Pedregosa, M.; Córdoba, F.; Villalba, J.; González-

Reyes, J.

2003. Zonal changes in ascorbate and hydrogen peroxide contents, peroxidase and ascorbate- related enzyme lactivities in onion roots. Plant Physiol. 131: 647-706.

Elloumi, O.; Ghrab, M.; Kessentini, H.; Ben Mimoun, M.

2013. Chilling accumulation effects on performance of pistachio trees cv. Mateur in dry and warm area climate. Scientia Horticulturae 159: 80-87.

Erez, A.

1995. Means to compensate for insufficient chilling to improve bloom and leafing. Acta Hort. 395: 81-95.

Erez, A.; Yablowitz, Z.; Korchinski, R.

1993. High density planting for protected cultivation of fruit crops; plastic cover for peach and nectarine. Acta Hort. 349: 129-132.

Ghrab, M.; Mimoun, M.; Masmoudi, M.

2014. Chilling trends in a warm production area and their impact on flowering and fruiting of peach trees. Scientia Horticulturae 178: 87-99.

Halaly, T.; Pang, X.; Batikoff, T.; Crane, O.; Keren, A.

2008. Similar mechanism might be triggered by alternative external stimuli that induce dormancy release in grape buds. Planta 228: 79-88.

Ben Mohamed, H.; Vadel, M.; Geuns, J.; Khemira, H.

2010. Biochemical changes in dormant grapevine shoot tissues in response to chilling: Possible role in dormancy release. Scientia Horticulturae 124: 440-447.
Ben Mohamed, H.; Vadel, M.; Geuns, J.; Khemira, H.

2012. Carbohydrate changes during dormancy release in Superior Seedless grapevine cuttings following hydrogen cyanamide treatment. Scientia Horticulturae 140: 19-25.

Hauagge, R.; Cummins, J.

1991. Relathionship among indices for the end bud dormancy in apple cultivars and related Malus species. J. Amer. Hort. Sci. 116 (1): 107-115.

Hung, K.; Kao, C.

2004. Hydrogen peroxide is necessary for abscisic acidinduced senescence of rice leaves. J. of Plant Physiol. (161): 1347-1357.

Kuroda, H.; Sugiura, T.; Sugiura, H.

2005. Effect of hydrogen peroxide on breaking endodormancy in flower buds of Japanese pear (Pyrus pyrifolia Nakai). J. Japan. Soc. Hort. Sci. 74 (3): 255-257.

Kuroda, H.; Sugiura, T.; Ito, D.

2002. Changes in hydrogen peroxide content in flower buds of Japanese pear (Pyrus pyrifolia Nakai) in relation to breaking of endodormancy. J. Japan. Soc. Hort. Sci. 71 (5): 610-616.

Lang, G.

1987. Dormancy: A new universal terminology. HortScience 22: $817-820$.

Lanino, $\mathrm{M}$.

2004. Antecedentes climáticos de la Estación Experimental Canchones. Revista de Agricultura del Desierto. 3: 57-68.

Londo, J.; Johnson, L.

2014. Variation in the chilling requirement and budburst rate of wild Vitis species. Enviromental Botany 106: 138-147.

Marquat, C.; Vandammme, M.; Gendraud, N.; Pétel, G.

1999. Dormancy in vegetative buds of peach: relation between carbohydrate absortion potentials and carbohydrate concentration in the during dormancy and its release. Scientia Horticulturae. 79: 151-162.

Meitha, K.; Konnerup, D.; Colmer, T.; Considine, J.; Foyer, C.; Considine, $\mathrm{M}$.

2015. Spatio-temporal relief from hypoxia and production of reactive oxygen species during bud burst in grapes (Vitis vinifera). Annals of Botany. 116: 703-711.

Noctor G.; Foyer, C.

1998. Ascorbate and glutathione keeping active oxygen under control. Annu. Rev. Plant Physiol. Plant Mol. Biol. 49: 249-279.

Nir, G.; Shulman, Y.; Fanberstein, L.; Lavee, S.

1986. Changes in the activity of catalase (EC 1.11.1.6) in relation to the dormancy of grapevine (Vitis vinifera L.) buds. Plant Physiol. 81: 1140-1142.

Parada, F.; Noriega, X.; Dantas, D.; Bressan-Smith, R.; Pérez, F. 2016. Differences in respiration between dormant and nondormant buds suggest involvement of ABA in development of endodormancy in grapevines. Journal in Plant Physiology 201: 71-78.

Pérez, F.; Lira, W.

2005. Possible role of catalase in post-dormancy bud break in grapevines. J. of Plant Physiol. 162: 301-308.

Pérez, F.; Ormeño, J.; Reynaert, B.; Rubio, S.

2008a. Utilización del modelo dinámico para evaluar el frío invernal en una localidad de clima templado y otra subtropical de Chile. Chilean J. Agric. Res. 68: 198-206. 
Pérez, F.; Rubio, S.; Ormeño, J.

2007. Is erratic bud-break in grapevines grown in warm winter areas related to disturbances in mitochondrial respiratory capacity and oxidative metabolism. Functional Plant Biology 34: 624-632.

Pérez, F.; Vergara, R.; Rubio, S.

2008b. $\mathrm{H}_{2} \mathrm{O}_{2}$ is envolved in the dormancy-breaking effect of hydrogen cyanamide in grapevine buds. Plant Growth Regul. 55: 149-155.

Pinto, M.; Lira, W.; Ugalde, $\mathrm{H}$.

2008. Fisiología de la latencia en yemas de vid: hipótesis actuales. Uvas Rústicas: Cultivo e Processamento en Regioes Tropicais. Universitaria Gráfica \& Editora Rua Mathias Schmdt. Em: Conceicao, Fracaro, de Souza, pp. 136-158.

Poblete, I.; Lanino, M.; Pinto, M.; Riquelme, A.

2009. Efecto de cianamida hidrogenada y peróxido de hidrógeno sobre la brotación y cosecha en vid (Vitis vinifera L.) en accesión Canchones, en condiciones de la pampa del Tamarugal, región de Tarapacá. Simiente 79: 37-46.

Poblete, I.; Pinto, M.; De Andrés, M. T.; Hinrichsen, P.

2011. Genetic characterization of old grapevines colleted in oases of the Atacama desert. Chilean J. Agric. Res. 71 (3): 476-482.

Richardson, E.; Seeley, S.; Walker, D.

1974. A model for estimating the completion of rest for Redhaven and Elberta peach trees. HortScience 9 (4): 331-332.
Rubio, S.; Donoso, A.; Pérez, F.

2014. The dormancy-breaking stimuli "chilling, hipoxia and cyanamide exposure" up-regulate the expression of $\alpha$-amylase genes in grapevine buds. Journal of Plant Physilogy. 171: 373-381.

Rubio, S.; Dantas, D.; Bressan-Smith, R.; Pérez, F.

2016. Relationship between endodormancy and cold hardiness in grapevine buds. J. Plant Growth Regul 35: 266-275.

Taylor, J.; Paris, R.; Loveys, B.; Notodimedjo, S.

1984. Changes in endogenous in apple during bud burst induced by defoliation. Plant Growth Regul. 2: 117-134.

Vergara, R.; Pérez, F.

2010. Similarities between natural and chemically induced bud-endodormancy release in grapevine Vitis Vinifera L. Scientia Horticulturae 125: 648-653.

Vergara, R.; Parada, F.; Rubio, S.; Pérez, F.

2012. Hipoxia induce $\mathrm{H}_{2} \mathrm{O}_{2}$ production and activates antioxidant defense system in grapevine buds through mediation of $\mathrm{H}_{2} \mathrm{O}_{2}$ and ethylene. Journal of Experimental Botany 63 (11): 4123-4131.

Warrington, I.; C. Stanley, S.

1986. The influence of pre-and post-budbreak temperatures on flowering in kiwi fruit. Acta Hort. 175: 103-107.

Zhi-You, Y.; Xian-Li, L.; Ling-Hao, L.; Xing-Guo, H.; Tian-Li, 2003. Effects of temperature and several chemicals on metabolic changes during dormancy release in NJ72 nectarine. Agricultural Sci. in China 2 (5): 549-555. 